\title{
Self-sizing radiofrequency ablation balloon for eradication of Barrett's esophagus: results of an international multicenter randomized trial comparing 3 different treatment regimens
}

Kamar Belghazi, MD, ${ }^{1}$ Roos E. Pouw, MD, PhD, ${ }^{1}$ Arjun D. Koch, MD, PhD, ${ }^{2}$ Bas L. A. M. Weusten, MD, $\mathrm{PhD},{ }^{3}$ Erik J. Schoon, MD, PhD, ${ }^{4}$ Wouter L. Curvers, MD, PhD, ${ }^{4}$ Annieke W. Gotink, MD, ${ }^{2}$ Nahid Mostafavi, PhD, ${ }^{5}$ Rehan J. Haidry, MD, PhD, ${ }^{6}$ Oliver Pech, MD, PhD, ${ }^{7}$ Jacques J. G. H. M. Bergman, $\mathrm{MD}, \mathrm{PhD}$,

Raf Bisschops, MD, $\mathrm{PhD}^{8}$

Amsterdam, Rotterdam, Nieuwegein, Eindhoven, the Netherlands; London, UK; Regensburg, Germany; Leuven, Belgium

Background and Aims: Recently, the 360 Express radiofrequency ablation balloon catheter (360 Express, Med- tronic, Minneapolis, Minn, USA) has replaced the traditional system for circumferential radiofrequency ablation (RFA) of Barrett's esophagus (BE). The aim was to compare 3 different ablation regimens for the 360 Express.

Methods: An international multicenter noninferiority randomized controlled trial was conducted in which pa- tients with a BE $(2-15 \mathrm{~cm})$ with dysplasia or early cancer were randomly assigned to the standaxd $\left(110 \mathrm{~J} / \mathrm{cm}^{2}\right.$-clean- $\left.1 \quad 10 \mathrm{~J} / \mathrm{cm}^{2}\right)$, simple-double $(210 \mathrm{C}$ $\mathrm{J} / \mathrm{cm}^{2}$-no clean), or simple-single ablation regimen (1 $10 \mathrm{~J} / \mathrm{cm}^{2}$-no clean). The primary outcome was the percentage endoscopically visual BE regression at 3 months. Sec- ondary outcomes were procedure time, adverse events, and patient discomfort.

Results: Between September 2015 and October 2017, 104 patients were enrolled. The simpledouble ablation arm was closed prematurely because of a $21 \%$ stenosis rate. The trial continued with the standard (n Z 37) and simple-single arm ( $\mathrm{n} \mathrm{Z} \mathrm{38).} \mathrm{Both} \mathrm{arms} \mathrm{were}$ comparable at baseline. Noninferiority of the simple-single arm could not be demonstrated: $\mathrm{BE}$ regression was $73 \%$ in the simple-single arm versus $85 \%$ in the standard arm; the median difference was $13 \%$ (95\% confidence interval, $5 \%-23 \%)$. The procedure time was significantly longer in the standard arm (31 vs 17 minutes, $P<.001)$. Both groups were comparable with regard to adverse events and patient discomfort.

Conclusions: This randomized trial shows that circumferential RFA with the 360 Express using the simple-double ablation regimen results in an unacceptable high risk of stenosis. Furthermore, the results suggest that a single ablation at $10 \mathrm{~J} / \mathrm{cm}^{2}$ results in inferior $\mathrm{BE}$ regression at 3 month $\boldsymbol{x}$ We therefore advise using the standard ablation regimen $(1 \quad 10 \mathrm{~J} / \mathrm{cm}-$ clean-1 $10 \mathrm{~J} / \mathrm{cm}$ ) for treatment of $\mathrm{BE}$ using the 360 Express. (Clinical trial registration number: NTR5191.) (Gastrointest Endosc 2019;90:415-23.)

\section{INTRODUCTION}

Radiofrequency ablation (RFA), with previous endo- scopic resection (ER) of visible lesions when present, has been shown to be effective and safe in the treatment of dysplastic Barrett's esophagus (BE). ${ }^{1-4}$ Most patients first undergo circumferential balloon-based RFA, followed by subsequent focal RFA sessions. Traditionally, circumferen- tial RFA of BE was performed using the Barrx360 system (Medtronic, Minneapolis, Minn, USA). The Barrx360 pro- cedure started with sizing the esophageal inner diameter (EID) at multiple levels. Based on these measurements, an appropriately sized ablation catheter, with a $3-\mathrm{cm}$-long electrode, was selected and used to ablate the Barrett's segment. For the traditional system, 2 ablation regimens could be applied: the standard regimen consisting of $2 \mathrm{ab}$ - lations at $12 \mathrm{~J} / \mathrm{cm}^{2}$ with a cleaning phase in between, and a simplified regimen in which the same zone was sequentially ablated twice without a cleaning step. A previous random- ized trial showed that both ablation regimens were equally effective and safe; however, the simplified regimen was significantly faster and required fewer introductions of the endoscope and ablation catheter. ${ }^{5}$ Disadvantages of the traditional Barrx 360 system were that it required separate introduction of the sizing catheter and subsequently the ablation catheter, and that the entire Barrett's segment was treated with an ablation catheter with a fixed diameter. To 
overcome these disadvantages, the traditional system was recently replaced by the 360 Express RFA balloon catheter (360 Express, Medtronic, Minneapolis, Minn, USA). The 360 Express consists of a 4-cmlong electrode that is wrapped around a balloon and has the ability to self-adjust to the esophageal lumen ensuring optimal tissue contact during each ablation. A recent pilot study showed that circumferen- tial RFA with the 360 Express using the standard ablation regimen $\left(12 \mathrm{~J} / \mathrm{cm}^{2}\right.$-clean-12 $\left.\mathrm{J} / \mathrm{cm}^{2}\right)$ leads to a decreased pro- cedure time but maintains efficacy when compared with the traditional system. ${ }^{6}$ However, after one 360 Express treatment, pronounced esophageal scarring was observed in $23 \%$ of the patients. Although no symptomatic stenosis was seen after a single treatment session, $10 \%$ of the patients did develop a stenosis requiring dilation after subsequent focal RFA treatment.

In the current study, we sought to evaluate the optimal ablation regimen for the 360 Express. Because the new 360 Express balloon adjusts to the EID and has a longer elec- trode, it may be a more effective but also potentially a more aggressive ablation tool, and therefore the energy density settings for the 360 Express were lowered from $12 \mathrm{~J} / \mathrm{cm}^{2}$ to $10 \mathrm{~J} / \mathrm{cm}^{2}{ }^{6}$ We evaluated the standard regimen at $10 \mathrm{~J} / \mathrm{cm}^{2}$, a simplified regimen with 2 ablations at $10 \mathrm{~J} / \mathrm{cm}^{2}$ without a cleaning phase, and a third arm in which a single hit at $10 \mathrm{~J} / \mathrm{cm}^{2}$ was performed. The aim of this study was to compare these 3 different regimens for circumferential RFA of BE using the 360 Express. 


\section{METHODS}

\section{Study design and setting}

This study was designed as a multicenter noninferiority randomized controlled trial comparing 3 arms. The trial was conducted in 5 tertiary referral centers for the manage- ment of early Barrett's neoplasia in the Netherlands and Belgium: Amsterdam University Medical Center, location AMC, Amsterdam; Catharina Hospital, Eindhoven; Erasmus MC, University Medical Center, Rotterdam; St. Antonius Hospital, Nieuwegein; and UZ Gasthuisberg, Leuven.

An independent data and safety monitoring board (DSMB), consisting of 2 gastroenterologists with experi- ence in using RFA and a statistician, was assigned to monitor the safety of the participants. Safety stopping rules were created to guide the DSMB in decision making.

\section{Patient selection}

Patients were eligible if they met all of the following in- clusion criteria: age 18 to 85 years; $\mathrm{BE}$ between 2 and $15 \mathrm{~cm}$ at baseline (before any ER); and biopsy-proven low-grade dysplasia, high-grade dysplasia, or cancer confirmed by an expert pathologist. In the case of previous ER for early cancer or visible lesions, the resection had to be limited to $<2 \mathrm{~cm}$ in length and $<50 \%$ of the circumference. Patients were excluded if they had any of the following exclusion criteria: previous ablation treatment; significant esophageal stenosis preventing passage of a diagnostic endoscope or any previous dilatation for esophageal steno- sis; presence of esophageal varices; anti-coagulant therapy (apart from aspirin or nonsteroidal anti-inflammatory drugs) that could not be discontinued; uncorrectable he- mostatic disorders; an interval of $>6$ months between the last high-resolution endoscopy with biopsies and RFA treat- ment; an interval $<6$ weeks between ER and RFA; residual cancer in biopsy specimens obtained after ER and before RFA; and in the case of previous ER, specimen(s) showing positive vertical resection margins, deep submucosal inva- sion $(>\mathrm{T} 1 \mathrm{sm} 1)$, poorly or undifferentiated cancer, or lymphatic/vascular invasion. In addition, patients had to

give informed consent.

\section{Randomization}

Patients were randomly assigned in a 1:1:1 ratio to treat- ment with the standard $(1 \quad 10 \mathrm{~J} / \mathrm{cm}$-clean$110 \mathrm{~J} / \mathrm{cm})$, simple-double (2 $10 \mathrm{~J} / \mathrm{cm}$-no clean), or simple-single (1 $10 \mathrm{~J} / \mathrm{cm}$-no clean) ablation regimen. Randomization of eligible patients was performed on site by thxlocal study coordinator during the study endoscopy after eligibility of the patient was coxfirmed bythe endoscopist. The random- ization sequence was computer generated (ALEA randomi- zation software, FormsVision, Abcoude, The Netherlands) and was stratified by previous ER. The allocation sequence was concealed from the study personnel enrolling patients by using random block sizes of 3,6 , and 9 .

The 2 primary outcome assessors (R.H., O.P.) were blinded to the allocation. Patients and study personnel were aware of the allocated treatment arm.

\section{Study device}

All patients were treated with the 360 Express. The 360 Express consists of a 4-cm-long, 360-degree electrode array that is wrapped around a self-adjusting balloon that can range in diameter from 18 $\mathrm{mm}$ to $31 \mathrm{~mm}$ (Fig. 1). Further details were described previously. ${ }^{6}$ The Barrx360 express catheters used in this investigator-initiated study were supplied by Medtronic (Minneapolis, Minn, USA).

\section{Interventions}

Work-up endoscopy before inclusion. Within 6 months before inclusion (as part of standard clinical care), a high-resolution endoscopy was performed during which the entire Barrett's segment was carefully inspected. If pre- sent, visible abnormalities were removed using ER. The (re- sidual) Barrett's segment was sampled by taking random biopsy samples according to the Seattle protocol $(4 \mathrm{Q} / 2 \mathrm{~cm})$. Study procedure: 360 Express RFA. All procedures were performed with the patients under monitored anes- thesia or conscious sedation. The Barrett's segment was cleaned with water. The procedure started with inspection of the Barrett's segment using high-resolution white-light endoscopy (WLE) and optical chromoendoscopy (eg, narrow-band imaging, blue-light imaging, or Fuji Intelligent Chromo Endoscopy, Fuji, Tokyo, Japan). When no visible abnormalities and no significant stenosis were present, pa- tients were randomized. Still images were taken of every centimeter of the Barrett's segment using WLE and optical chromoendoscopy. After placement of a stiff guidewire, the 360 Express was introduced, followed by reintroduc- tion of the endoscope to position the catheter at the 
upper end of the Barrett's segment using visual guidance.

For the standard ablation regimen (1X $10 \mathrm{~J} / \mathrm{cm}$-clean- 1 X $10 \mathrm{~J} / \mathrm{cm})$, the entire Barrett's segment was ablated $(10 \mathrm{~J} / \mathrm{cm} 2$ at $300 \mathrm{~W})$ working from proximal to distal, reposi- tioning the catheter under visual control. After the first ablation pass, the endoscope and ablation catheter were removed. The catheter was cleaned and the endoscope with a distal attachment cap attached to its tip was reintro- duced to wipe off the coagulum from the ablated segment. Then the guidewire was replaced, the endoscope was removed, and the catheter and endoscope were reintro- duced to repeat the ablation.

For the simple-double ablation regimen (2 $10 \mathrm{~J} / \mathrm{cm}$ - no clean), immediately after the first ablatiox $(10$ $\mathrm{J} / \mathrm{cm}^{2}$ at $300 \mathrm{~W}$ ), a second ablation was performed on the same zone without cleaning. The balloon was advanced distally to ablate subsequent zones under visual control with a double ablation in an identical way.

For the simple-single ablation regimen (1 $10 \mathrm{~J} / \mathrm{cm}$-no clean), the entire Barrett's segment was ablated with 1 hit of energy $\left(10 \mathrm{~J} / \mathrm{cm}^{2}\right.$ at $\left.300 \mathrm{~W}\right)$ undervisual control, working from proximal to distal, using visual repositioning without cleaning.

Postablation care. All patients received a maintenance high-dose proton pump inhibitor throughout the treatment period. For most patients, this medication was supplemented with ranitidine $300 \mathrm{mg}$ at bedtime and su- cralfate suspension 3 times a day for a period of 2 weeks after the RFA procedure.

Follow-up after treatment. Three months after the 360 Express procedure, post-treatment follow-up endos- copy was performed. Still images with high-resolution WLE and optical chromoendoscopy were again obtained at 1-cm intervals throughout the extent of the original Bar- rett's segment. Further treatment and follow-up were per- formed according to standard clinical guidelines. In general, repeat RFA treatment was performed at 3- monthly intervals until complete endoscopic eradication of all Barrett's tissue was achieved.

\section{Outcomes}

Definitions of all outcome parameters are summarized in Table 1 . The primary outcome was the mean percentage of endoscopically visual surface regression of $\mathrm{BE}$ epithelium at 3 months, as scored by 2 independent endoscopists (R.H. and O.P.) blinded to the allocated ablation regimen. The secondary outcomes were adverse events, procedure time, and patient discomfort after RFA treatment. Patient discomfort consisted of 2 parameters: retrosternal pain directly after the procedure and the cumulative daily discomfort score (for 30 days after the procedure).

\section{Sample size}

We assumed that there would be no difference in the per- centage of $B E$ regression among the 3 ablation protocols. ${ }^{1}$

Therefore, a noninferiority design was used comparing the standard protocol with either of the simplified protocols. We assumed that a difference of $15 \%$ or more in percentage BE regression would be clinically relevant. Using a significance level of .0125 (Bonferroni correction because of 2 comparisons), a noninferiority margin of 0.15 , a standard deviation (SD) of 0.17 , and power of 0.9 , we calculated that 36 patients would be required per arm when taking a $10 \%$ dropout rate into consideration. Therefore, we sought to enroll a total of 108 patients. For the sample size calculation, statistical software $\mathrm{R}$ version

3.4.3 SampleSize4ClinicalTrial package was used.

\section{Changes to study design after commencement of the trial}

The DSMB recommended early closure of the simple- double arm because of an unacceptable high risk of adverse events. After approval of the Medical Ethical Com- mittee (MEC), the trial continued with the 2 remaining arms and patients were randomized in a 1:1 fashion.

\section{Statistical methods}

For descriptive statistics, the mean (SD) was used in cases of normal distribution of variables, and the median (interquartile range [IQR]) was used for variables with a skewed distribution. Categorical variables are presented as frequencies with percentages. For the primary outcome, the median difference between the percentage Barrett's regression of the standard ablation arm and the simple- single arm was calculated together with the corresponding $95 \%$ confidence interval (CI). Noninferiority of the simplesingle arm could be concluded when the prespecified non- inferiority margin was not included in the CI, whereas infe- riority could be concluded when the prespecified noninferiority margin was included in 
the CI. For the secondary outcomes a Student $t$ test or Wilcoxon rank sum test was used for the comparison of continuous variables with a normal distribution or skewed distribution, respectively.

Categorical variables were compared using the chi- squared or Fisher exact test. For the cumulative discomfort score, the duration of discomfort symptoms was estimated using Kaplan-Meier analysis. The event in the analysis was "free of discomfort symptoms," which was defined as a cumulative discomfort score of 0 . The 2 groups were compared with a log rank test. A 2 -sided $P$ value $<.05$ was considered significant in the analyses. All analyses were performed on the modified intention-to-treat (m-ITT) population. Data analysis was performed using SPSS statistical software package (version 24; SPSS Inc, Chi- cago, Ill).

Post hoc analysis. Poor response was defined as 50\% endoscopically visible BE regression after one 360 Express treatment, as scored by the 2 blind\&d endo- scopists mentioned previously. In a post hoc analysis, we compared the presence of 4 known baseline independent predictors associated with a poor response between the groups: active reflux esophagitis, ER regeneration with $\mathrm{BE}$, esophageal narrowing pre-RFA, and months of dysplasia pre-RFA.

\section{Ethical considerations}

The study protocol was reviewed and approved by the MEC of Amsterdam UMC and the UZ Gasthuisberg Leuven and approved by the local MECs in the remaining 3 partici- pating centers. Written informed consent was obtained from all participants. The trial was registered at www. trialregister.nl (registration number NTR5191). All authors had access to the study data and reviewed and approved the final manuscript.

\section{RESULTS}

\section{Patients}

Between September 14, 2015, and October 23, 2017, a to- tal of 104 patients were enrolled (Fig. 2). In February 2017, inclusion in the simple-double ablation arm was stopped prematurely because of an unexpected high risk of stenosis. At that moment, 28 patients $(24$ men, median C4M5 BE, pre- vious ER in 12 patients) were already included in this arm (Table 2). The interim analysis showed that 6 patients (21\%; 95\% CI, 10\%-39\%) developed a stenosis. Of these 6 patients, 4 developed a severe stenosis requiring 12 dilations and 2 incision therapies, 8 dilations, 7 dilations, 4 dilations and 1 incision therapy, respectively. One patient had a moderate stenosis requiring 4 dilations, and one stenosis was graded as mild (1 dilation). Figure 3 shows endoscopic images of the severe stenoses. The DSMB recommended early closure of the simple-double arm because of the frequency and the severity of the stenoses (median, 6 dilations; range, 1-12). Table 3 shows the outcomes of the simple-double regimen arm.

The trial continued with the 2 remaining arms. Thirty- seven patients were allocated to the standard ablation regimen and 39 to the simple-single regimen. One patient received treatment that was not in concordance with randomization and was therefore excluded. The remaining 75 patients were included in the m-ITT population. For 3 patients, no data were available on the primary endpoint, and these patients could therefore not be included in the primary analysis (Fig. 2). Both groups were similar at baseline (Table 2).

\section{Primary outcome: Barrett's regression at 3 months}

The median Barrett's regression was $85 \%$ (IQR, 75\%- 94\%) in the standard arm versus $73 \%$ (IQR, $46 \%-83 \%$ ) in the simple-single arm, based on blinded assessor scores. In $79 \%$ (57 of 72 ) of the cases, there was $<10 \%$ agreement between the 2 assessors. For 2 cases, there was $>30 \%$ dif- ference in regression score, and a consensus diagnosis was made.

The median difference between both groups was $13 \%$ (95\% CI, 5\%-23\%). Because the prespecified noninferiority margin of $15 \%$ is included in the $95 \% \mathrm{CI}$ of the median dif- ference, we concluded that the percentage BE regression was inferior in the simple-single arm.

In a post hoc analysis, the proportion of patients showing a poor response was significantly lower in the standard arm compared with the simple-single arm: 3 of $35(9 \%)$ versus 12 of $36(33 \%)$ $(P Z$.013). The 4 known baseline predictors for a poor response are presented in Table 2. No statistically significant difference was found in these baseline predictors between the standard and the simple-single arm: active reflux esophagitis ( $P$ Z.32), esophageal narrowing $(P Z$.25), ER scar 
regeneration with Barrett's epithelium $(P Z$.70), and months of dysplasia before RFA treatment (PZ.34).

\section{Secondary outcomes}

Adverse events. There was no significant difference in the number of adverse events between the groups: $5(14 \%)$ in the standard arm versus $4(11 \%)$ in the simple-single arm (P Z .74) (Table 3). In the standard arm, 4 patients had a minor laceration during the 360 Express ablation (mild adverse event). In 1 patient the laceration occurred after inflation of the balloon and further 360 Express treatment could not be performed. All 4 lacerations required no treatment. One patient died due to an unrelated cause 10 weeks after the 360 Express treatment. Because this event occurred before the patient had his first follow-up endoscopy and he was thus still in the study, we reported this as a serious adverse event. In the simple-single arm, all 4 adverse events were graded as mild. Three patients had a minor laceration dur- ing the 360 Express ablation without the need for treat- ment. The fourth patient was admitted for 1 night due to retrosternal pain. No stenoses were reported in the stan- dard or simple-single arm.

Procedure time. The median total procedure dura- tion was 31 minutes (IQR, 26-35 minutes) in the standard arm versus 17 minutes (IQR, 13-20 minutes) in the simple-single arm $(P<.001)$. The median ablation duration was also significantly longer in the standard arm compared with the simplesingle arm: median, 18 minutes (IQR, 15- 22 minutes) versus 6 minutes (IQR, 5-8 minutes) $(P<.001)$. Patient discomfort after RFA treatment. The me- dian pain score for retrosternal pain directly after the pro- cedure was 2 in both groups $(P Z$.4). In the standard ablation group, 31 patients completed the diary for the cu- mulative discomfort score 30 days after the procedure versus 30 in the simple-single arm. There was no statisti- cally significant difference in the duration of discomfort symptoms. Patients in both arms were free of symptoms after a median of 10 days ( $P$ Z .11) (Fig. 4).

\section{DISCUSSION}

This international multicenter randomized controlled trial aimed to evaluate the optimal ablation regimen for circumferentialRFA ofBE using the 360 Express. We found 
that a simple-single hit $\left(10 \mathrm{~J} / \mathrm{cm}^{2}\right)$ regimen was less effec- tive and did not meet the criteria for noninferiority in com- parison with a standard regimen using 2 hits of $10 \mathrm{~J} / \mathrm{cm}^{2}$ with a cleaning step. In addition, a double $10 \mathrm{~J} / \mathrm{cm}^{2}$ hit regimen resulted in an unacceptable high stricture rate and should therefore not be used.

The trial was originally designed to compare 3 different ablation regimens: the standard ablation regimen, the simple-double regimen, and the simple-single regimen. However, during the trial, a stenosis rate of $21 \%$ was found in the simple-double ablation arm and, after studying all the stenoses cases, the DSMB advised early closure of this arm. In the literature, the stenosis rate for RFA varies between $0 \%$ and $14 \%,{ }^{8}$ but this stenosis rate is based on the entire treatment period (so after additional ablation sessions). The $21 \%$ stenosis rate found in our study was after 1 simple-double ablation procedure and was there- fore much higher than one would expect based on the literature. More importantly, the severity of the stenosis in our study was worse than anticipated based on previous studies and experience. Most patients required more than 4 dilations and/or incision therapy, whereas the stenoses reported in the literature are generally mild and well treat- able with a median of 1 to 2 dilations. A previous random- ized trial showed that the simple-double regimen did not result in more stenoses compared with the standard regimen when using the traditional Barrx 360 system. ${ }^{5}$ So, why does the simple-double regimen result in an increased risk of severe stenosis when the 360 Express is used? First, when using the Barrx360 system, a 3-cm-long ablation cath- eter was chosen based on the smallest measured EID. Therefore, with the former system, the apposition of the electrode to the esophageal mucosa was less effective and often obtained by additional suctioning. In contrast, the new 360 Express balloon adjusts to the EID, creating a maximal and optimal contact between the electrode and the esophageal wall. In addition, the ablation catheter has a longer electrode of $4 \mathrm{~cm}$ and delivers more energy to the esophagus than the traditional system. Second, when using the standard regimen, the esophageal wall has time to "cool down" during the cleaning step. Furthermore, the time until the next ablation allows submucosal edema induced by the first ablation to have its protective effect on the submucosa and deeper wall layers. When the cleaning step is omitted, as is the case in the simpledouble regimen, the ablation damage may be deeper, resulting in more fibrosis.

After closure of the simple-double arm, the trial was temporarily put on hold and a safety signal was published to inform readers of the increased stenosis risk that is associated with the simple-double ablation regimen. ${ }^{9}$ Furthermore, the study design was adjusted, and after approval of the MEC, the trial was continued with the 2 remaining arms.

The final results of our trial show that circumferential ablation with the 360 Express using the single hit regimen results in inferior endoscopically visible BE surface regres- sion at 3 months compared with the standard regimen. In addition, the number of patients showing a poor BE regres- sion response at 3 months was significantly higher in the simple-single arm. A previously published prospective study identified 4 independent predictors for a poor initial response 3 months after circumferential RFA. ${ }^{7}$ We performed a post hoc analysis and both groups were comparable with regard to the presence of the above- mentioned predictors. Therefore, it is unlikely that the pa- tients in the simple-single arm were more prone to have a poor regression response.

One may argue that the standard ablation regimen re- sults in a longer procedure time and that a single ablation with a higher energy setting $\left(12 \mathrm{~J} / \mathrm{cm}^{2}\right)$ may be a valid alter- native ablation protocol. However, we believe that the dif- ference in $\mathrm{BE}$ regression between the 2 groups is most likely to be explained by the number of hits (1 vs 2). A regimen with 2 hits of energy is probably more effective in ablating all Barrett's mucosa because there is another chance to hit areas that were not optimally ablated during the first ablation pass or to ablate skipped zones during the second pass. We therefore believe that increasing the en- ergy setting from 10 to $12 \mathrm{~J} / \mathrm{cm}^{2}$ will not significantly in- crease the efficacy of a single hit ablation regimen. 
When interpreting the endoscopically visible $\mathrm{BE}$ regres- sion results, one should keep in mind that additional focal ablation sessions will be performed and that a difference of Barrett's regression at 3 months does not necessarily affect the overall efficacy measured at the end of the treatment period. However, one may argue that less residual $\mathrm{BE}$ mu- cosa during follow-up will require less-extensive additional RFA treatment, thus exposing the patient to a lower risk of adverse events than when more extensive additional abla- tion is required.

Strengths ofthis studyincludethe stratified randomized design of the study and the blinded primary outcome assessment, increasing the internal validity. In addition, to improve generalizability, we included patients with a wide range in $\mathrm{BE}$ length and performed the study in mul- tiple centers. Furthermore, a DSMB commission was as- signed to monitor the safety of the patients. Finally, the perspective of patients was taken into account by adding patient reported measurements to the outcomes.

This study has several limitations that need to be ad- dressed. First, the study was conducted in 5 tertiary referral centers and treatment was performed by Barrett expert en- doscopists. Therefore, one should be careful about extrap- olating the results to general practice. However, we believe that treatment of early Barrett's neoplasia should be limited to expert centers with dedicated endoscopists who are trained in the detection of early neoplastic lesions and experienced in performing endoscopic treatment. Sec- ond, only 61 of the 74 patients completed the 30-day discomfort diary. However, the response rate was compa- rable in both groups, so it is not likely that the suboptimal response rate has introduced bias because of selective nonresponse. Third, 4 patients could not be included in the primary analysis. One patient was excluded from the study after randomization because he was accidently treated with the wrong ablation regimen, and this may have introduced bias in favor of the alternative hypothesis. In 3 patients, no data on the primary outcome were avail- able. Two of these patients were assigned to the standard arm; this resulted in 35 analyzable patients in this arm instead of the required 36 patients based on the initial sample size calculation. However, this did not influence the conclusion of the primary analysis because there was still $>90 \%$ of power to assess the noninferiority. After the simple-double arm was closed, the sample size was recalcu- lated with an adjustment of the significance level to .025 because the Bonferroni correction could be omitted because only 2 arms were compared. According to the new sample size calculation there would be 94\% power when 36 patients were included per arm.

In conclusion, the results of this international random- ized controlled trial are clinically relevant because many en- doscopists who have been using the simple-double ablation regimen successfully with the traditional RFA catheter have copied this regimen when using the 360 Express. However, the results of our trial show that the simple-double regimen

is associated with an unacceptable risk of severe stenosis. Furthermore, the results of this study suggest that a single ablation at $10 \mathrm{~J} / \mathrm{cm}^{2}$ when using the 360 Express results in inferior BE regression at 3 months. We therefore advise us- ing the standard ablation regimen (1 $10 \mathrm{~J} / \mathrm{cm}$-clean-1 $\left.10 \mathrm{~J} / \mathrm{cm}^{2}\right)$ for the 360 Express in treatment of $\mathrm{BE}$, as is rec- ommended in the instructions for use. 


\section{REFERENCES}

1. Pouw RE, Wirths K, Eisendrath $P$, et al. Efficacy of radiofrequency abla- tion combined with endoscopic resection for Barrett's esophagus with early neoplasia. Clin Gastroenterol Hepatol 2010;8:23-9.

2. van Vilsteren FG, Pouw RE, Seewald S, et al. Stepwise radical endoscopic resection versus radiofrequency ablation for Barrett's oesophagus with high-grade dysplasia or early cancer: a multicenter multicenter trial. Gut 2011;60:765-73.

3. Phoa KN, Pouw RE, Bisschops R, et al. Multimodality endoscopic eradi- cation for neoplastic Barrett oesophagus: results of an European multi- center study (EURO-II). Gut 2016;65:555-62.

4. Haidry RJ, ButtMA, Dunn JM, etal. Improvement over time in outcomes for patients undergoing endoscopic therapy for Barrett's oesophagus- related neoplasia:6-yearexperience from the first500 patients treated in the UK patient registry. Gut2015;64:11929.

5. van Vilsteren FG, Phoa KN, Alvarez Herrero L, et al. Circumferential balloon-based radiofrequency ablation of Barrett's esophagus with dysplasia can be simplified, yet efficacy maintained, by omitting the cleaning phase. Clin Gastroenterol Hepatol 2013;11:491-8.e1.

6. Belghazi K, Pouw RE, Sondermeijer CMT, et al. A single step sizing and RFA catheter for circumferential ablation of BE: results of a pilot study. United European Gastroenterol J 2018;6:990-9.

7. van Vilsteren FG, Alvarez Herrero L, Pouw RE, et al. Predictive factors for initial treatment response after circumferential radiofrequency ablation for Barrett's esophagus with early neoplasia:a prospective multicenter study. Endoscopy 2013;45:516-25.

8. Chadwick G, Groene O, Markar SR, et al. Systematic review comparing radiofrequency ablation and complete endoscopic resection in treating dysplastic Barrett's esophagus: a critical assessment of histo- logic outcomes and adverse events. Gastrointest Endosc 2014; 79:718-31.

9. Pouw RE, Bergman JJ. Safety signal for the simple-double ablation regimen when using the Barrx ${ }^{\mathrm{TM}} 360$ express RFA balloon catheter.

Gastroenterology 2017;15:614. 

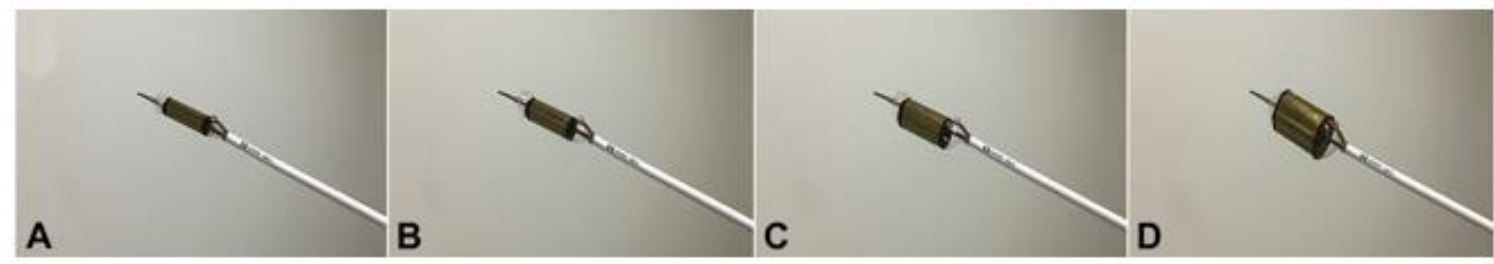

Figure 1. Wrap/unwrap electrode design of the 360 Express radiofrequency ablation balloon catheter. A, The balloon catheter is fully wrapped. B, C, By inflating the balloon the catheter unwraps. D, The balloon is inflated and the catheter is fully unwrapped.

\section{TABLE 1. Definitions of outcome parameters}

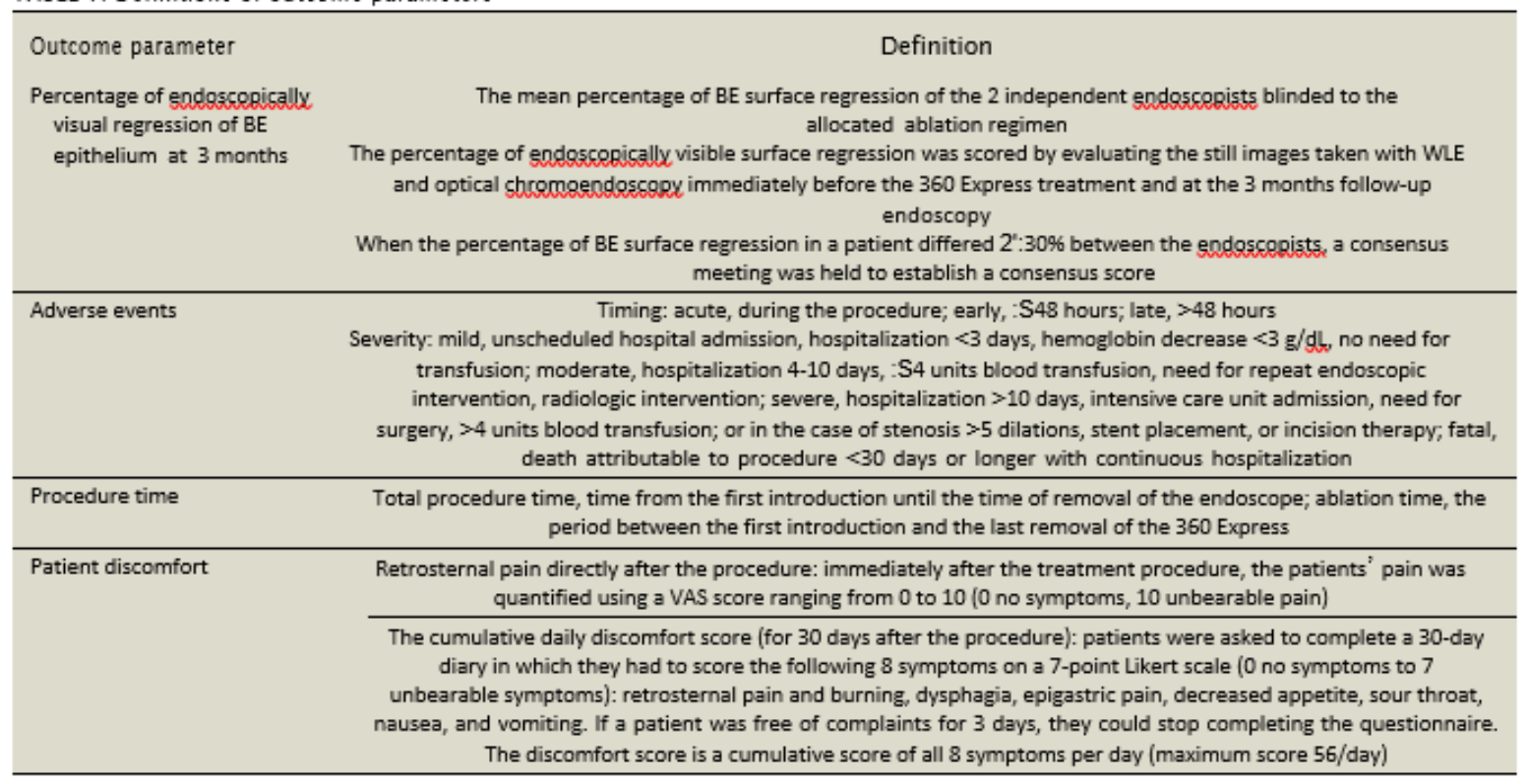

BE, Barrett's esophagus; WLE, white-light endoscopy; VAS, visual analog scale.

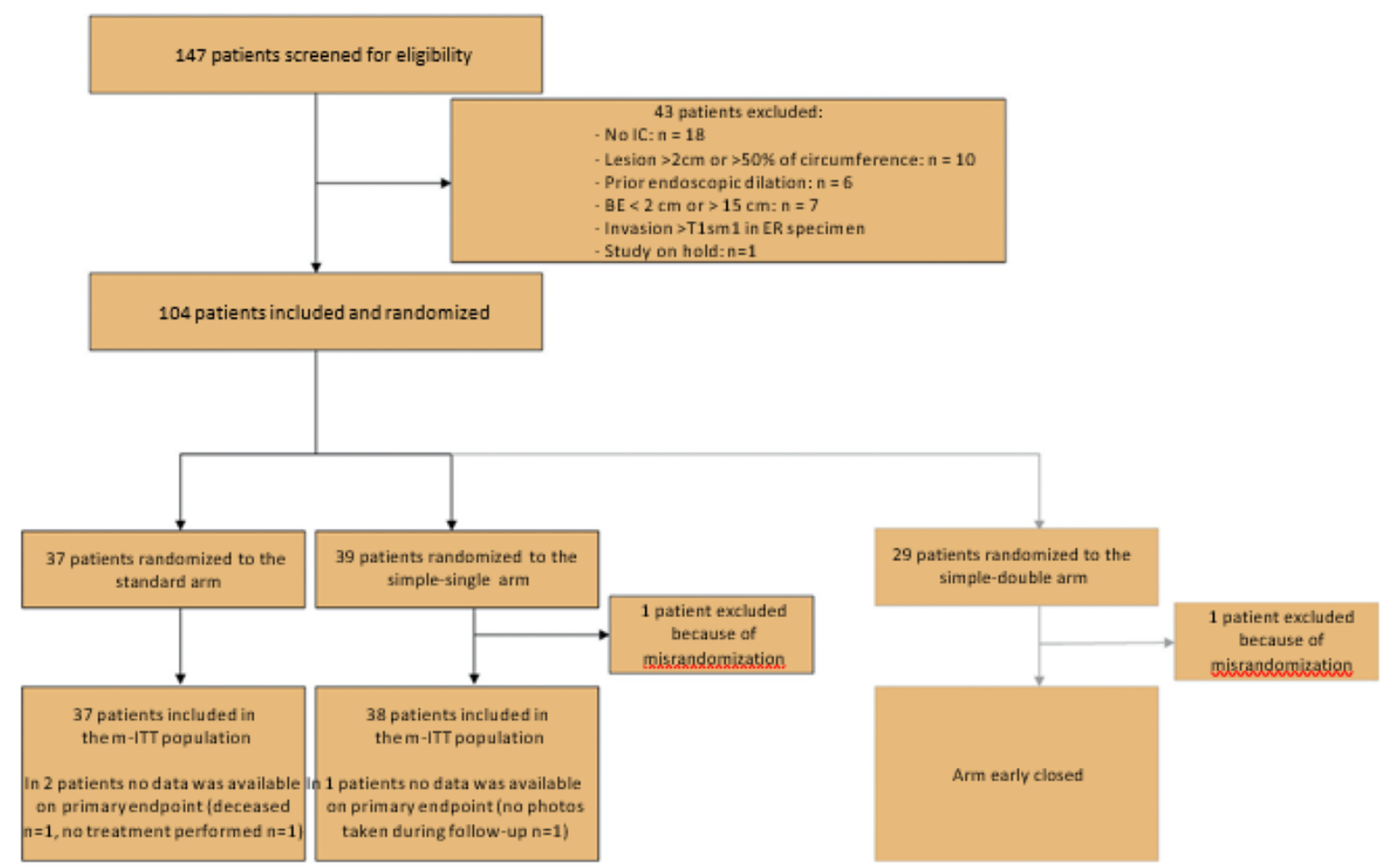

'igure 2. Flowchart of the enrollment. IC, informed consent; $m$-ITT, modified intention-to-treat analysis; $B E$, Barrett's esophagus; and ER, endoscopic esection. 
TABLE 2. Baseline and clinical characteristics

\begin{tabular}{|c|c|c|c|}
\hline Characteristics & Standard arm & Simple-single arm & $\begin{array}{l}\text { Simple-double arm } \\
\text { (arm early closed) }\end{array}$ \\
\hline No. of patients & 37 & 38 & 28 \\
\hline Male:female & $29: 8$ & 28:10:00 & 24:04:00 \\
\hline Age (years), median (IQR) & $65(61-72)$ & $67(63-70)$ & $67(61-70)$ \\
\hline $\mathrm{BE}(\mathrm{cm})$, median (IQR) & $\mathrm{C} 4(2-7), \mathrm{M7}(5-10)$ & $\mathrm{C6}(2-7), \mathrm{M} 8(5-10)$ & C4 (1-5), M6 (4-8) \\
\hline Active reflux esophagitis at baseline, $\mathrm{n} \quad(\%)^{*}$ & $0(0)$ & $1(3)$ & $0(0)$ \\
\hline Esophageal narrowing at baseline, $n(\%)^{*}$ & $2(5)$ & $5(13)$ & $0(0)$ \\
\hline \multicolumn{4}{|l|}{$\begin{array}{l}\text { Worst overall histologic diagnosis before } \\
\text { treatment (biopsies or ER specimens), } n(\%)\end{array}$} \\
\hline Low-grade dysplasia & $11(30)$ & $15(39)$ & $11(39)$ \\
\hline High-grade dysplasia & $18(49)$ & $15(39)$ & $11(39)$ \\
\hline Early cancer & $8(21)$ & $8(21)$ & $6(21)$ \\
\hline ER before RFA, n (\%) & $15(41)$ & $16(42)$ & $12(43)$ \\
\hline \multicolumn{4}{|l|}{ Worst histology of ER specimens, $\mathrm{n}$} \\
\hline Nendysolastic BE & 2 & 0 & 1 \\
\hline Low-grade dysplasia & 0 & 2 & 2 \\
\hline High-grade dysplasia & 5 & 6 & 3 \\
\hline Early cancer & 8 & 8 & 6 \\
\hline ER scar regeneration with Barrett's epithelium, n (\%) & $4(27)$ & $6(38)$ & $1(8)$ \\
\hline \multicolumn{4}{|l|}{$\begin{array}{l}\text { Worst overall histologic diagnosis before } \\
\text { RFA treatment, after ER if applicable, } \mathrm{n} \text { (\%) }\end{array}$} \\
\hline Nendysplastic BE & $3(8)$ & $5(13)$ & $5(18)$ \\
\hline Low-grade dysplasia & $18(49)$ & $18(47)$ & $17(61)$ \\
\hline High-grade dysplasia & $16(43)$ & $15(40)$ & $6(21)$ \\
\hline Months of dysplasia before RFA, median (IQR)* & $6(3-9)$ & $7(4-11)$ & $5(4-12)$ \\
\hline \multicolumn{4}{|l|}{ Portablation care } \\
\hline High-dose PPI p ranitidine $\mathrm{p}$ succalfate, $n$ (\%) & $22(59)$ & $30(79)$ & $23(82)$ \\
\hline High-dose PPI p ranitidine, n (\%) & $15(41)$ & $8(21)$ & $5(18)$ \\
\hline
\end{tabular}

IQR, Interquartile range; $B E$, Barrett's esophagus; $E R$, endoscopic resection; $R F A$, radiofrequency ablation; $P P I$, proton pump inhibitor.

"Knawn independent baseline predictors for a paor initial regression response. ${ }^{7}$ 


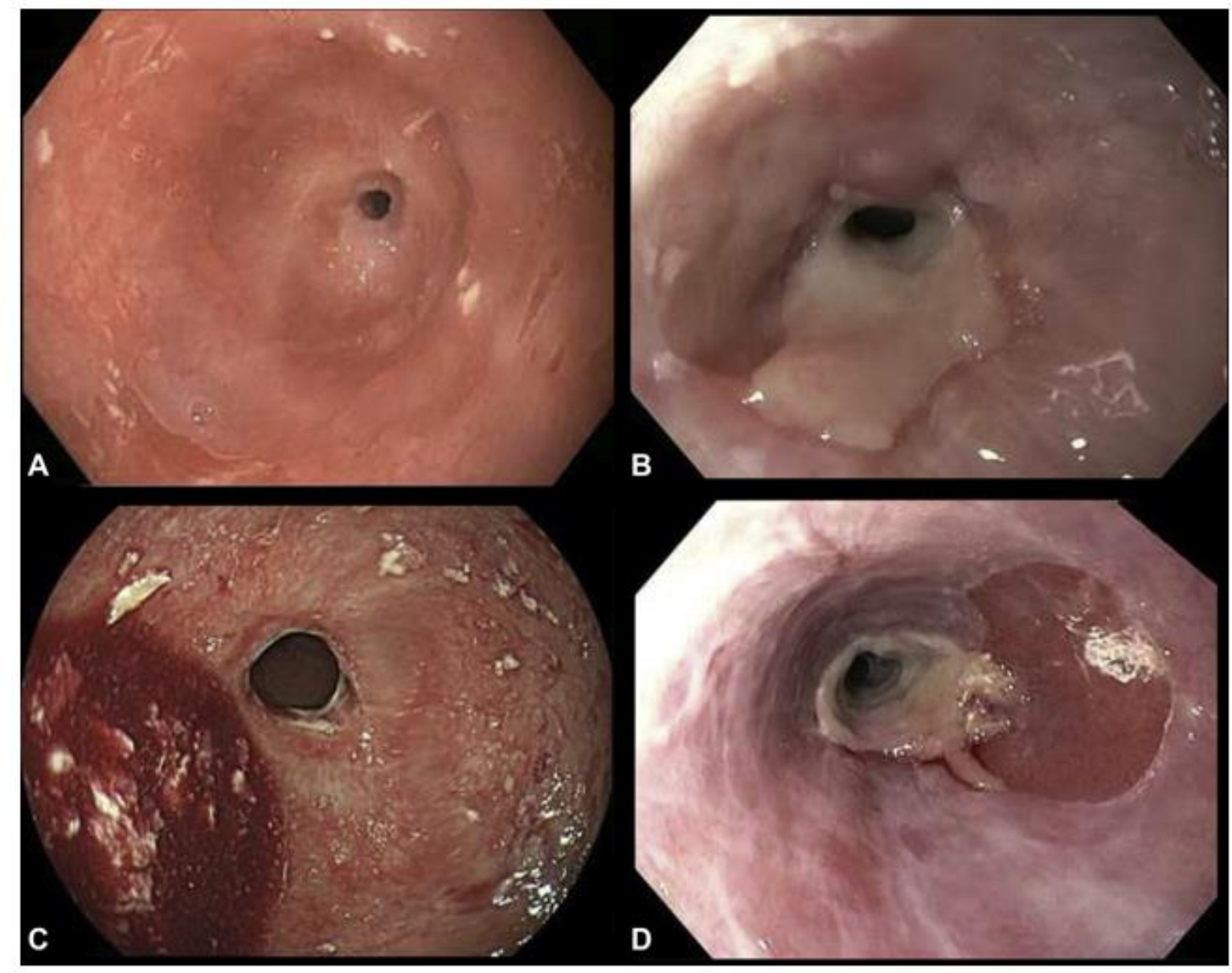

Figure 3. Endoscopic images of 4 patients [A-D] with a severe stricture after a single 360 Express treatment using the simple-double regimen.

TABLE 3. Outcomes

\begin{tabular}{|c|c|c|c|}
\hline Ablation regimen & Standard arm & Simple-single arm & $\begin{array}{l}\text { Simple-double arm } \\
\text { (arm closed early) }\end{array}$ \\
\hline No. of patients & 37 & 38 & 28 \\
\hline Percentage BE regression at 3 months, median (IQR) & $85(75-94)$ & $73(46-83)$ & $88(81-93)$ \\
\hline Procedure duration (minutes), median (IQR) & $31(26-35)$ & $17(13-20)$ & $17(14-20)$ \\
\hline Ablation duration (minutes), median (IQR) & $18(15-22)$ & $6(5-8)$ & $6(5-9)$ \\
\hline Overall adverse event rate, $n$ (\%) & $5(14)$ & $4(11)$ & $7(25)$ \\
\hline Stricture requiring an intervention, $\mathrm{n}(\%)$ & $0(0)$ & $0(0)$ & $6(21)$ \\
\hline Minor laceration, n (\$) & $4(11)$ & $3(8)$ & $0(0)$ \\
\hline Other adverse events, n ( 96 ) & Unrelated death: 1 (3) & Retrosternal pain and fever: 1 ( 3 ) & Near collapse: 1 (4) \\
\hline
\end{tabular}

$B E$, Barrett's esophagus; IQR, interquartile range. 


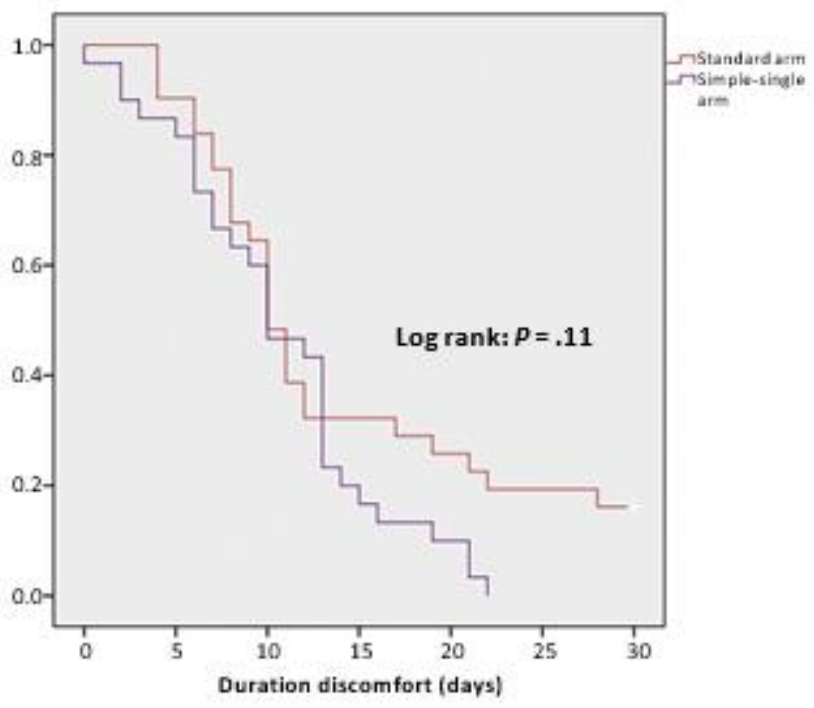

Figure 4. Kaplan-Meier plot showing the duration of discomfort इxmptoms 30 days after the 360 Express treatment. 